\title{
Sarcopenia as a Novel Prognostic Factor in the Patients of Primary Localized Gastrointestinal Stromal Tumor
}

\section{He Song}

The first hospital of China medical univeristy https://orcid.org/0000-0002-0775-9701

Ming Dong ( $\nabla$ dongming@cmu.edu.cn )

the first hospital of China medical university

\section{Research article}

Keywords: sarcopenia, gastrointestinal stromal tumor, skeletal muscle index, survival

Posted Date: April 22nd, 2020

DOl: https://doi.org/10.21203/rs.3.rs-22159/v1

License: (c) (i) This work is licensed under a Creative Commons Attribution 4.0 International License.

Read Full License 


\section{Abstract}

\section{Background}

Sarcopenia predicts poor prognosis of a variety of gastrointestinal malignancies. However, there is a lack of study on the association between skeletal muscle index (SMI) and the prognosis of gastrointestinal stromal tumor (GIST). The aim of this study is to develop a novel nomogram based on sarcopenia for GIST patients to predict overall survival (OS)

\section{Methods}

SMI was measured by computed tomography scan of 107 patients who underwent resection for primary localized gastrointestinal stromal tumor (GIST). Sarcopenia was defined by cutoff values for SMI as $40.1 \mathrm{~cm}^{2} / \mathrm{m}^{2}$ and $39.8 \mathrm{~cm}^{2} / \mathrm{m}^{2}$ using optimum stratification for males and females respectively. Factors were included in the nomogram were specified by univariate and multiple Cox proportional hazard analysis. Concordance index (C-index) and calibration curves were conducted to measure the discrimination and accuracy of the nomogram. The utility of the nomogram was assessed by the decision curve analysis (DCA).

\section{Results}

Twenty-eight (26.2\%) of 107 patients were sarcopenic. Sarcopenia was correlated significantly with BMI, albumin, female sex, resection style, mitotic index, rupture status, survival. Sarcopenia was significantly related to decreased overall survival $(p=0.003)$. The nomogram including sarcopenia status, resection style and mitotic index had an excellent discrimination with C-index 0.794. The calibration curves represented a good accordance between the actual observation and nomogram prediction for overall survival. Decision curve analysis illustrated that the nomogram was helpful in clinic .

\section{Conclusions}

We developed a nomogram based on sarcopenia to predict overall survival after resection of GISTs which is an effective and favorable prognostication tool.

\section{Background}

Gastrointestinal stromal tumor (GIST) is the most common mesenchymal neoplasm occurring from the gastrointestinal tract(1). Worldwide, the prevalence of GIST is 4.3-22 per million per year, with approximately mid $60 \mathrm{~s}$ of age and equal gender distribution(2). Before millennium, traditional remedy, for instance, chemotherapy and radiation were not effective on GIST. Identification of the driver mutations in c-KIT and platelet-derived growth factor receptor a (PDGRF-a) further led to the successful targeted therapy of tyrosine kinase inhibitor (TKI)-imatinib (3). Despite advances in treatment modalities, radical resection with TKI treatment remains mainstay for GIST. Especially, the GIST patients with the intermediate and high risk are recommended for the TKI treatment postoperatively. Risk stratification 
might differentiate with patients who need TKI treatment and those who do not. Therefore, a more precise and refined stratification system is truly needed to manage postoperative therapeutics.

In 2002, Fletcher (4)firstly developed the predicting system for GIST, presently described as the NIH criterion comprising two factors (mitotic index and tumor size). In 2006, the Armed Forces Institute of Pathology (AFIP) criterion (5) added tumor location in comparison to NIH criterion. In 2008, the National Institute of Health $(\mathrm{NIH})$ risk stratification system was modified in addition to tumor rupture status compared to AFIP. This modified NIH criterion has been widely accepted because it is more easier to apply than the AFIP criteria, and subsequent Memorial Sloan-Kettering Cancer Center (MSKCC) nomogram (6)in 2009 Joensuu's contour maps (7)in 2012. All the GIST recruited in this cohort were confirmed as the intermediate and high risk category based on modified $\mathrm{NIH}$ classifications (tumor size, mitotic rate, location, and rupture).

Sarcopenia is an age-related disorder defined by accelerating depletion in muscle mass and decline in strength and physical performance. Recently, sarcopenia has been regarded as the predictor of poorer (disease free survival) DFS or (overall survival) OS in many malignances (8-10). Sarcopenia frequently occurs in the disease of chronic disorder such as cancer. In addition, aging could easily induce muscle atrophy(11), and GIST likely occur in the mid 60's (2). So, we raised a hypothesis that there might be a relationship between sarcopenia and outcome of GIST.

There are various methods to evaluate the sarcopenia, such as muscle mass, muscle strength and physical performance. Measured value of muscular mass area by a cross-sectional computed tomography (CT) image at the level of the third lumbar vertebra (L3), namely skeletal muscle index (SMI) are generally accepted(12). Due to SMI based on the preoperative imaging, sarcopenia might be a potential biomarker influencing the prognosis of GIST. However, currently, there were few reports on the role of sarcopenia in patients with GIST.

\section{Method}

\subsection{Study design}

This study was a single-center retrospective analysis. We evaluated 107 patients pathologically proven to be GIST with intermediate or high risk category who underwent surgical resection in the department of general surgery, the first hospital of China medical university from February 2013 to February 2019. All the patients were followed up postoperatively by telephone interview, outpatient visits and China's native app-wechat and all surviving patients were followed up for more than 6 years. This research was approved by Ethics committee of the first hospital of China medical university.

The inclusion criteria were as follows: (1) more than 18 years; (2) abdominal computed tomography (CT) scans available within a month before surgery; (3) primary localized neoplasm; (4) patients received TKI treatment for at least 6 months postoperatively (5) intermediate and high risk category according to modified NIH classifications. The exclusion criteria were: (1) distant metastasis;;(2) insufficient 
clinicopathologic data; (3) pretreatment therapy (e.g. preoperative oral administration of imatinib for down stage)

\subsection{CT valuation}

Every patient of CT-image valuation was performed using ImageJ software.. Skeletal muscle volume was evaluated to use the CT image for all the eligible patients. A transverse CT examination at the third lumbar vertebra (L3) of the single $5 \mathrm{~mm}$-slice was assessed. The muscles in the $\mathrm{L} 3$ region-including internal and external obliques, transverse and rectus abdominus, psoas, quadratus lumborum, and erector spinae were analyzed.

L3 skeletal muscle index ( $\left.\mathrm{L} 3 \mathrm{SMI}, \mathrm{cm}^{2} / \mathrm{m}^{2}\right)$ is calculated as follows: cross-sectional areas measurements of the muscle $\left(\mathrm{cm}^{2}\right)$ at the $L 3$ divided by the height.

Our own sex-specific cut-off values for the L3 SMI were established at which the survival difference was most significant, we used optimum stratification to define the SMI cut-off value $39.8 \mathrm{~cm} 2 / \mathrm{m} 2$ for women and $40.1 \mathrm{~cm} 2 / \mathrm{m} 2$ for men. SMI below the defined cutoff value was described as sarcopenia. The method of optimum stratification has been previously described in literature (13) to separate sarcopenic patients and non-sarcopenic patients.

\subsection{Statistical analysis}

The characteristics of the 2 groups (sarcopenia and non-sarcopenia) were compared. Univariable analysis was performed using $\chi^{2}$ test, the Mann-Whitney $U$ test for categorical variables and independentsample $t$ test for continuous variables. The Kaplan-Meier method was used to estimate OS. Variables with $p<0.05$ in univariable analysis were included in multivariable analysis. A Cox proportional-hazards regression analysis was performed. The predictive performance of nomogram related to survival was assessed by $\mathrm{C}$-index. The utility of the nomogram was evaluated by decision curve analysis (DCA). $P$ value of less than 0.05 was considered significant. All statistical analyses were performed with the Statistical Package for Social Sciences (SPSS) 24.0 for Windows (Chicago, Illinois) and R software (version 3.5·0).

\section{Results}

\section{1 clinical parameters of GIST patients}

Among 107 enrolled patients, 28 (26.2\%) were classified as the sarcopenia group and 79 (73.4\%) into the non-sarcopenia group. The clinical characteristics (Table 1) between the two groups were compared. The mean value of $\mathrm{BMI}$ and serum albumin in the non-sarcopenia group was higher than in the sarcopenia group ( $P=0.001 ; P=0.007)$. Significant difference in resection style $(p=0.034)$, mitotic index $(p=0.02)$, rupture status $(p=0.007)$, survival status $(p=0.001)$ were found. Age, hemoglobin, gender, tumor site, tumor size and pathological type were not observed to differ significantly in two groups. 
By univariate analysis, sarcopenia $(p=0.003)$, resection style $(p=0.001)$ and mitotic index $(p=0.005)$ were significant predictors of OS. They were also further conducted in the multivariate analysis. sarcopenia $(p=0.016)$, resection style $(p=0.036)$ and mitotic index $(p=0.044)$ were still identified as the independent prognostic factor for OS in GIST patients. (shown in the table 2)

At the time of final follow-up (Feb, 2019), 18 of 107 patients (16.8\%) were dead, and the median follow-up for was 25.4 months. The median overall survival of patients with sarcopenia defined by SMI was significantly lower ( 40.6 months; $95 \% \mathrm{Cl}, 31.6-49.6)$ than those with normal skeletal muscles $(63.8$ months; $95 \% \mathrm{Cl}, 57.6-69.8 ; \mathrm{P}=0.0011$ ) (shown in the Fig. 1A). The patients undergoing incomplete resection represented the poorer survival compared to the patients with complete resection $(p=0.00015$, shown in the Fig. 1B). The patients with high mitotic index (ख10HPF) represented significantly worst survival ( $p=0.0017$, shown in the Fig. $1 C)$

\subsection{Establishment of nomogram}

In the multivariable COX proportion hazard regression models, 3 factors including sarcopenia status, resection style and mitotic index were significantly associated with mortality of GIST. Therefore, nomogram of three factors was established (shown in the Fig. 2) to predict the probability of 3 year- and 5 year- 0 S

The associated concordance index of nomogram (c-index) was 0.794 (95\% Cl 0.747-0.841), which indicated that $79.4 \%$ of the probability of individual mortality would be correctly predicted by the nomogram model. The calibration curves represented high consistency in the prognostic value of 3 yearand 5 year-OS (Fig. $3 A+B)$.

\subsection{Clinical use of DCA curve analysis}

The decision curve analysis (shown in the FIG 4) elucidated that the nomogram was feasible to make clinical valuable decision.

\section{Discussion}

Our research showed a comprehensive analysis from clinicopathological data and CT scans to explore the survival outcomes in the Chinese population with GIST following surgery. Preoperative sarcopenia defined by SMI was associated with poor survival. CT has a high degree of validity in assessing body composition and is regarded as the gold standard method for estimating muscle $(10,14,15)$. To the best of our knowledge, this is the first research indicating that impaired effects of sarcopenia for the survival in patients with GIST worldwide.

Sarcopenia is a syndrome affecting innumerable people with cancers and is independent predictor of detrimental outcomes such as physical disability, poor quality of life, and reduced survival(16).However, the clinical definition of SMI remains inconclusive, the most widely used definitions were defined by 
Prado(13) in the western people. However, these definitions might not be applicable to Chinese GIST patients because $\mathrm{BMI}$ and physique differs greatly between eastern and western populations. Therefore, we used optimum stratification analysis to define the SMI cutoff. The incidence of sarcopenia with GIST patients was $26.2 \%(28 / 107)$ in our study is consistent with the previous study (17) with sarcopenic morbidity of $38.7 \%$ in the GIST patients.

Sarcopenic patients represented lower BMI and serum albumin. These two factors were associated with sarcopenia but not with the survival. Sarcopenia is considered to be a better predictive tool than BMI and albumin for survival. Albumin is a negative acute-phase protein that decreases in concentration with ongoing systemic inflammation, poor health, and malnutrition which lead to the decreased skeletal muscle mass(18), hence, lower albumin might be associated with low-SMI value to reflect the sarcopenic condition. This study is conformable with previous findings in other cancers $(19,20)$.

The high efficient predictive tool is indispensable for GIST patients. At present, a few influential predictive models for the prognosis of GIST have been designed. Miettinen (5)proposed that tumor size, mitotic rate, and tumor site can accurately predict the risk of GIST patients. Gold developed a nomogram for the recurrence free survival of patients with GIST patients after complete resections(6). This study established a new nomogram including resection style, mitotic index and sarcopenia. To prove the clinical validity, we evaluated whether the nomogram-assisted decisions would be beneficial to patient outcomes or not. The novel method of decision curve analysis demonstrated that if the threshold probability of a patient or doctor is $40 \%$, then probably 7.5 persons would benefit without detriment of others. Our nomogram also represent the reliable performance with high c-index of 0.794 . Our nomogram did not include other important clinical factors, for instance, the tumor size, tumor site and presence of rupture which have been reported to be correlated with the mortality of GIST patients(21-23). The possible reason might be some patient records affecting the data bias was excluded for insufficient clinical data. In the future study, we need the more clinical samples and validation set to prove our nomogram.

To reinforce the precision of tumor biological behavior prediction by only depending on resection style and mitotic counts, we integrated the new parameter sarcopenia by measurement of SMI into our nomogram. Preoperative and postoperative SMI are of equal importance. An interesting study(17) showed $63.6 \%$ of initially sarcopenic GIST patients became non-sarcopenic after 6 months of imatinib. This reversal might be explained by the drug's anti-tumor activity. Hence, clinicians should persist in nutritional guidance for the whole management of GIST patients. Meanwhile, patients are encouraged to exercise and receive appropriate nutritional treatment for muscle protein synthesis against sarcopenia(11, 24).

This study had several limitations. First, according to the European Working Group on Sarcopenia in Older People (EWGSOP), sarcopenia should be measured by the parameters of muscle mass, muscle strength and physical performance(25). We regarded only muscle mass as the definition of sarcopenia due to the retrospective design, and prospective study is further needed including more assessment tools for 
sarcopenia. Second, merely 107 patients were enrolled due to insufficient available clinical data and low incidence of GIST. Third, this was a single-institution study of small sample, and whether the results are feasible for other patient sets which needs further internal and external validation. Nevertheless, to best of our acknowledgement, this is the first study to establish a predicting model based on the preoperative sarcopenia of GISTs patients. More variables, for instance, inflammation index and gene detection could be incorporated in future.

\section{Conclusion}

In summary, we conducted a comprehensive analysis of preoperative sarcopenia associated with survival prognosis and established a new nomogram accurately predicting 3- and 5- survival for GIST patients. Thus, this study might be helpful for the physician to make better clinical evaluation.

\section{List Of Abbreviations}

Gastrointestinal stromal tumor, GIST; Overall survival, OS

\section{Declarations}

Ethics approval and consent to participate

The study was approved by the ethics committee of the first hospital of China medical university (no. 201711030). Patient consent was not required to review their medical records by the ethics committee of the first hospital of China medical university of its retrospective design, and exemption from informed consent did not adversely affect the health and rights of subjects. This study kept confidentiality of patient data and strictly complied with the Declaration of Helsinki and its later amendments or comparable ethical standards.

Consent for publication

Not applicable

Availability of data and materials

The datasets during and/or analyed during the current study available from the corresponding author on reasonable request.

Competing interests

We delcared no competing interests

Funding 
This work was supported in part by a grand-in aid for China Medical University Foundation for young key teachers (No. 3110118149), Natural science foundation of Liaoning province (No. 2019-BS-282)

Acknowledgements

Not applicable

\section{References}

1. Rubin BP, Heinrich MC, Corless CL. Gastrointestinal stromal tumour. The Lancet. 2007;369(9574):1731-41.

2. Soreide K, Sandvik OM, Soreide JA, Giljaca V, Jureckova A, Bulusu VR. Global epidemiology of gastrointestinal stromal tumours (GIST): A systematic review of population-based cohort studies. Cancer Epidemiol. 2016;40:39-46.

3. van Oosterom AT, Judson I, Verweij J, Stroobants S, di Paola ED, Dimitrijevic S, et al. Safety and efficacy of imatinib (STI571) in metastatic gastrointestinal stromal tumours: a phase I study. The Lancet. 2001;358(9291):1421-3.

4. Fletcher CD, Berman JJ, Corless C, Gorstein F, Lasota J, Longley BJ, et al. Diagnosis of gastrointestinal stromal tumors: A consensus approach. Human pathology. 2002;33(5):459-65.

5. Miettinen M, Lasota J. Gastrointestinal stromal tumors: Pathology and prognosis at different sites. Semin Diagn Pathol. 2006;23(2):70-83.

6. Gold JS, Gönen M, Gutiérrez A, Broto JM, García-del-Muro X, Smyrk TC, et al. Development and validation of a prognostic nomogram for recurrence-free survival after complete surgical resection of localised primary gastrointestinal stromal tumour: a retrospective analysis. The Lancet Oncology. 2009;10(11):1045-52.

7. Joensuu H, Vehtari A, Riihimäki J, Nishida T, Steigen SE, Brabec P, et al. Risk of recurrence of gastrointestinal stromal tumour after surgery: an analysis of pooled population-based cohorts. The Lancet Oncology. 2012;13(3):265-74.

8. Lou N, Chi CH, Chen XD, Zhou CJ, Wang SL, Zhuang CL, et al. Sarcopenia in overweight and obese patients is a predictive factor for postoperative complication in gastric cancer: A prospective study. Ejso-Eur J Surg Onc. 2017;43(1):188-95.

9. Choi MH, Yoon SB, Lee K, Song M, Lee IS, Lee MA, et al. Preoperative sarcopenia and post-operative accelerated muscle loss negatively impact survival after resection of pancreatic cancer. $\mathrm{J}$ Cachexia Sarcopenia Muscle. 2018;9(2):326-34.

10. Hopkins JJ, Reif R, Bigam D, Baracos VE, Eurich DT, Sawyer MM. Change in Skeletal Muscle Following Resection of Stage I-III Colorectal Cancer is Predictive of Poor Survival: A Cohort Study. World J Surg. 2019;43(10):2518-26.

11. Prado CM, Purcell SA, Laviano A. Nutrition interventions to treat low muscle mass in cancer. Journal of cachexia, sarcopenia and muscle. 2020. 
12. Tosato M, Marzetti E, Cesari M, Savera G, Miller RR, Bernabei R, et al. Measurement of muscle mass in sarcopenia: from imaging to biochemical markers. Aging Clin Exp Res. 2017;29(1):19-27.

13. Prado CMM, Liefers JR, McCargar LJ, Reiman T, Sawyer MB, Martin L, et al. Prevalence and clinical implications of sarcopenic obesity in patients with solid tumours of the respiratory and gastrointestinal tracts: a population-based study. Lancet Oncology. 2008;9(7):629-35.

14. Black D, Mackay C, Ramsay G, Hamoodi Z, Nanthakumaran S, Park KGM, et al. Prognostic Value of Computed Tomography: Measured Parameters of Body Composition in Primary Operable Gastrointestinal Cancers. Ann Surg Oncol. 2017;24(8):2241-51.

15. Kudou K, Saeki H, Nakashima Y, Edahiro K, Korehisa S, Taniguchi D, et al. Prognostic Significance of Sarcopenia in Patients with Esophagogastric Junction Cancer or Upper Gastric Cancer. Ann Surg Oncol. 2017;24(7):1804-10.

16. Nakashima Y, Saeki H, Hu Q, Tsuda Y, Zaitsu Y, Hisamatsu Y, et al. Skeletal Muscle Loss After Esophagectomy Is an Independent Risk Factor for Patients with Esophageal Cancer. Ann Surg Oncol. 2020;27(2):492-8.

17. Moryoussef F, Dhooge M, Volet J, Barbe C, Brezault C, Hoeffel C, et al. Reversible sarcopenia in patients with gastrointestinal stromal tumor treated with imatinib. J Cachexia Sarcopenia Muscle. 2015;6(4):343-50.

18. Santarpia L, Contaldo F, Pasanisi F. Nutritional screening and early treatment of malnutrition in cancer patients. J Cachexia Sarcopeni. 2011;2(1):27-35.

19. Yabusaki N, Fujii T, Yamada S, Suzuki K, Sugimoto H, Kanda M, et al. Adverse impact of low skeletal muscle index on the prognosis of hepatocellular carcinoma after hepatic resection. Int J Surg. 2016;30:136-42.

20. Visser M, Kritchevsky SB, Newman AB, Goodpaster BH, Tylavsky FA, Nevitt MC, et al. Lower serum albumin concentration and change in muscle mass: the Health, Aging and Body Composition Study. Am J Clin Nutr. 2005;82(3):531-7.

21. Dematteo RP, Gold JS, Saran L, Gonen M, Liau KH, Maki RG, et al. Tumor mitotic rate, size, and location independently predict recurrence after resection of primary gastrointestinal stromal tumor (GIST). Cancer. 2008;112(3):608-15.

22. Yanagimoto $Y$, Takahashi T, Muguruma K, Toyokawa T, Kusanagi H, Omori T, et al. Re-appraisal of risk classifications for primary gastrointestinal stromal tumors (GISTs) after complete resection: indications for adjuvant therapy. Gastric Cancer. 2015;18(2):426-33.

23. Rutkowski P, Nowecki ZI, Michej W, Debiec-Rychter M, Wozniak A, Limon J, et al. Risk criteria and prognostic factors for predicting recurrences after resection of primary gastrointestinal stromal tumor. Ann Surg Oncol. 2007;14(7):2018-27.

24. Fearon K, Strasser F, Anker SD, Bosaeus I, Bruera E, Fainsinger RL, et al. Definition and classification of cancer cachexia: an international consensus. Lancet Oncology. 2011;12(5):489-95.

25. Cruz-Jentoft AJ, Baeyens JP, Bauer JM, Boirie Y, Cederholm T, Landi F, et al. Sarcopenia: European consensus on definition and diagnosis: Report of the European Working Group on Sarcopenia in 
Older People. Age Ageing. 2010;39(4):412-23.

Figures
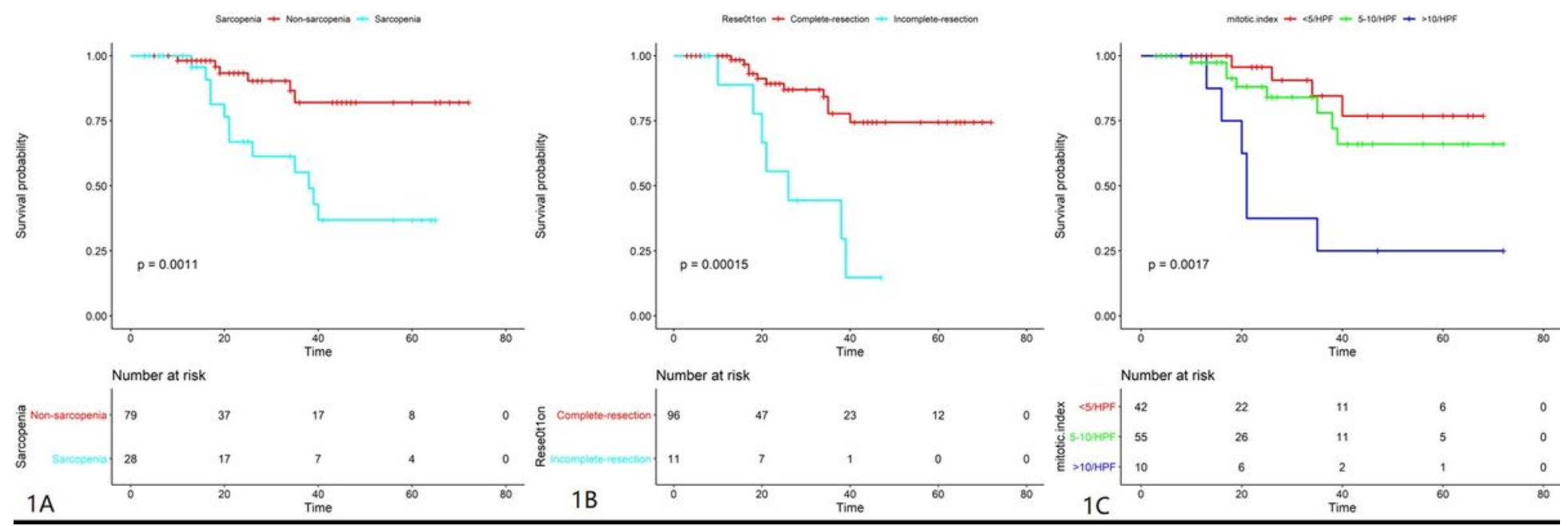

Figure 1

Kaplan-Meier curves of overall survival according to (A) sarcopenia status, (B)resection style and (C) mitotic index. Horizontal axis is calculated by month. 
Points

sarcopenia

resection style

mitotic.index

Total Points

3-year survival

5-year survival

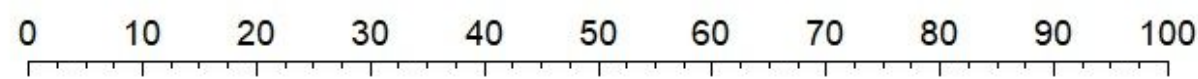

Sarcopenia

Non-sarcopenia

Incomplete-resection

Complete-resection

$<5 / \mathrm{HPF} \quad 5-10 / \mathrm{HPF}$

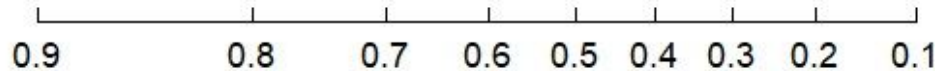

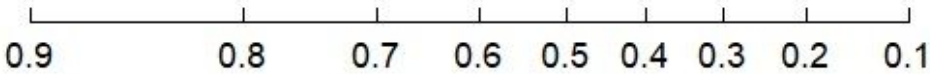

\section{Figure 2}

Nomogram for predicting GIST-related survival with sarcopenia status, resection style and mitotic index Note: The probability of each variable was added to converted into total score, and a vertical line was drawn on the total score to achieve the related probability of death.
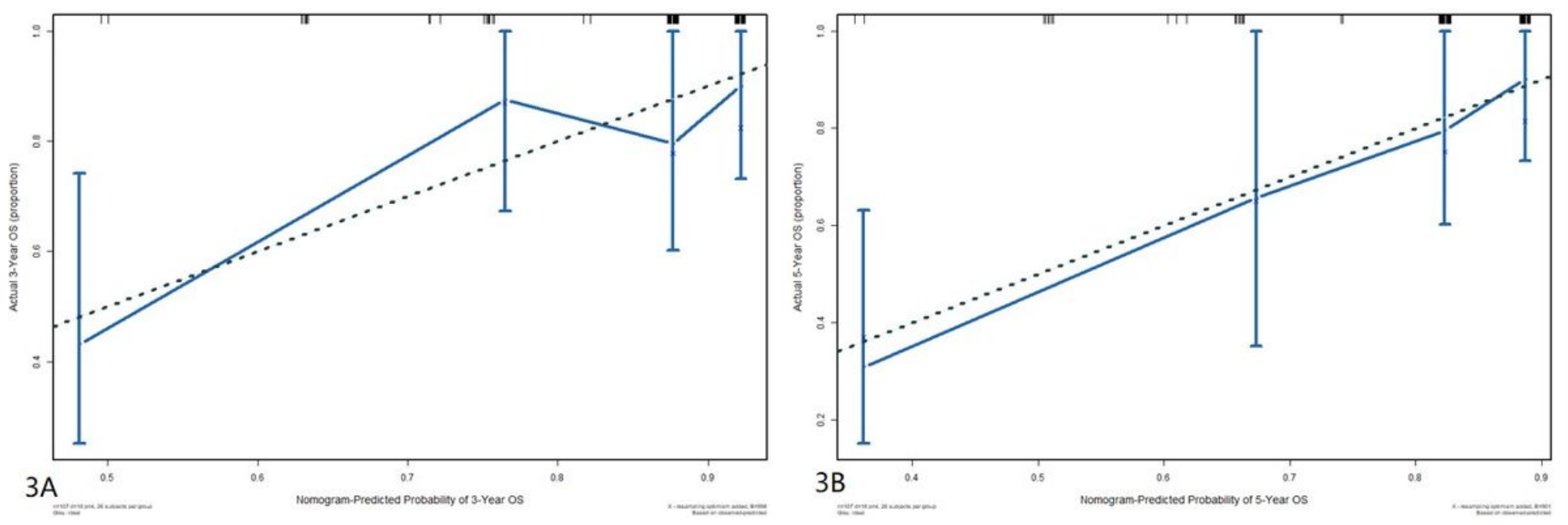

Figure 3 
Calibration curves of the prognostic nomogram for 3-year overall survival (A), 5-year overall survival (B). The $\mathrm{Y}$-axis indicates the observed overall survival of GIST while the X-axis indicates the estimated overall survival. The solid line demonstrates the ideal reference line that predicted GIST survival associated with the actual outcome whereas the dashed line demonstrates the prediction of nomogram. The closer alignment with the solid line represents the better performance is acquired.

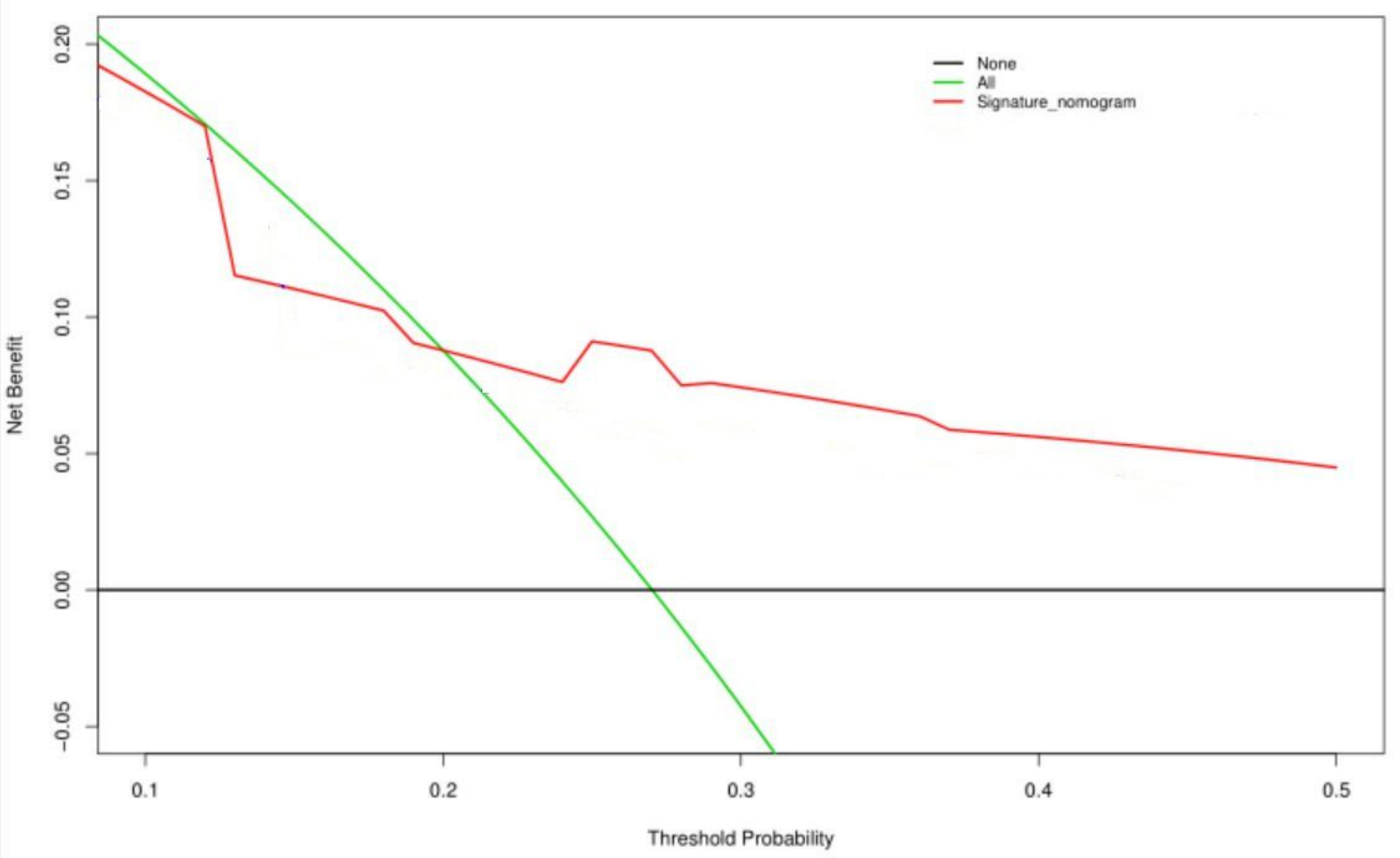

Figure 4

The Decision Curves Analysis curve of the predictive nomogram including three factors (sarcopenia status, resection style, mitotic index). The horizontal axis represents the threshold value, which is the where the expected benefit of treatment was equal to the expected benefit of avoiding treatment and the vertical axis represents adding up the true positive results and subtracting the false positive results. The nomogram (red line) has the high value due to the larger net benefit, 